\title{
Fixed Points of Difference Operator of Meromorphic Functions
}

\author{
Zhaojun $\mathrm{Wu}^{1}$ and Hongyan $\mathrm{Xu}^{2}$ \\ ${ }^{1}$ School of Mathematics and Statistics, Hubei University of Science and Technology, Xianning 437100, China \\ ${ }^{2}$ Department of Informatics and Engineering, Jingdezhen Ceramic Institute, Jingdezhen 333403, China
}

Correspondence should be addressed to Zhaojun Wu; wuzj52@hotmail.com

Received 28 August 2013; Accepted 24 October 2013; Published 19 January 2014

Academic Editors: M. Gandarias and Y. C. Shiah

Copyright (C) $2014 \mathrm{Z}$. Wu and $\mathrm{H}$. Xu. This is an open access article distributed under the Creative Commons Attribution License, which permits unrestricted use, distribution, and reproduction in any medium, provided the original work is properly cited.

Let $f$ be a transcendental meromorphic function of order less than one. The authors prove that the exact difference $\Delta f=f(z+$ 1) $-f(z)$ has infinitely many fixed points, if $a \in \mathbb{C}$ and $\infty$ are Borel exceptional values (or Nevanlinna deficiency values) of $f$. These results extend the related results obtained by Chen and Shon.

\section{Introduction and Main Results}

In this paper, we assume that the reader is familiar with the notations of frequency use in Nevanlinna theory (see [1-3]). Let $f(z)$ be a meromorphic function in the complex plane $\mathbb{C}$ and $a \in \mathbb{C}$. We use the notations $\sigma(f)$ to denote the order of $f(z), \lambda(f, a)$, and $\lambda(1 / f)$, respectively, to denote the exponent of convergence of zeros of $f(z)-a$ and poles of $f(z)$. Especially, if $a=0$, we denote $\lambda(f, 0)=\lambda(f)$. A point $z \in \mathbb{C}$ is called as a fixed point of $f(z)$ if $f(z)=z$. There is a considerable number of results on the fixed points for meromorphic functions in the plane; we refer the reader to Chuang and Yang [4]. It follows Chen and Shon [5]; we use the notation $\tau(f)$ to denote the exponent of convergence of fixed points of $f$ that is defined as

$$
\tau(f)=\limsup _{r \rightarrow \infty} \frac{\log N(r, 1 /(f-z))}{\log r} .
$$

Let $f$ be a transcendental meromorphic function in the complex plane $\mathbb{C}$. The exact differences $\Delta f$ are defined by $\Delta f=f(z+1)-f(z)$.

Recently, there are a number of papers (including [6-16]) focusing on the differences analogues of Nevanlinna's theory and its application on the complex difference equations. For the fixed points of the difference operator $\Delta f$, Chen and Shon have proved the following.
Theorem A (see [17]). Let $f$ be a transcendental entire function of order of growth $\sigma(f)=1$ and have infinitely many zeros with the exponent of convergence of zeros $\lambda(f)<1$. Then $\Delta f$ has infinitely many zeros and infinitely many fixed points.

When the order of $f$ is less than 1 , Chen and Shon have proved the following.

Theorem B (see [5]). Let $f$ be a transcendental meromorphic function of order of growth $\sigma(f) \leq 1$. Suppose that $f$ satisfies $\lambda(1 / f)<\lambda(f)<1$ or has infinitely many zeros (with $\lambda(f)=$ $0)$ and finitely many poles. Then $\Delta f$ has infinitely many fixed points and satisfies the exponent of convergence of fixed points $\tau(\Delta f)=\sigma(f)$.

A natural question is, letting $f$ be a transcendental meromorphic function of order of growth $\sigma(f)<1$, is there a similar result as that in Theorem B if $\lambda(1 / f) \geq \lambda(f)$ or $f$ has infinitely many zeros (with $\lambda(f)=0$ ) and infinitely many poles?

In this paper, we will prove the following theorem to answer the question.

Theorem 1 (main). Let $f$ be a transcendental meromorphic function of order of growth $\sigma(f)<1$ and $a \in \mathbb{C}$. Suppose that $f$ satisfies $\lambda(1 / f)<\sigma(f)$ and $\lambda(f, a)<\sigma(f)$. Then $\Delta f$ has infinitely many fixed points and satisfies the exponent of convergence of fixed points $\tau(\Delta f)=\sigma(f)$. 
From Theorem 1, we can get the following corollary.

Corollary 2. Let $f$ be a transcendental meromorphic function of order of growth $\sigma(f)<1$. Suppose that $f$ satisfies $\lambda(f) \leq$ $\lambda(1 / f)<\sigma(f)$. Then $\Delta f$ has infinitely many fixed points and satisfies the exponent of convergence of fixed points $\tau(\Delta f)=$ $\sigma(f)$.

In Theorem 1, we suppose that $f$ satisfies $\lambda(1 / f)<\sigma(f)$ and $\lambda(f, a)<\sigma(f)$. That is to say $\infty$ and $a$ are Borel exceptional values of $f$. If we suppose that $\infty$ and $a$ are Nevanlinna deficiency values of $f$, is there a similar result as that in Theorem B? In the following, we give Theorem 3 to answer this question.

Let $f(z)$ be a meromorphic function in the complex plane $\mathbb{C}$ and $a \in \mathbb{C}_{\infty}=\mathbb{C} \cup\{\infty\}$. Nevanlinna's deficiency of $f$ with respect to $a$ is defined by

$$
\delta(a, f)=1-\limsup _{r \rightarrow \infty} \frac{N(r, 1 /(f-a))}{T(r, f)} .
$$

If $a=\infty$, then one should replace $N(r, 1 /(f-a))$ in the above formula by $N(r, f)$. If $\delta(a, f)>0$, then $a$ is called a Nevanlinna deficiency value of $f$.

Theorem 3 (main). Let $f$ be a transcendental meromorphic function of order of growth $\sigma(f)<1$ and $a \in \mathbb{C}$. Suppose that $f$ satisfies $\delta(\infty, f)=1$ and $a$ is a Nevanlinna deficiency value of $f$. Then $\Delta f$ has infinitely many fixed points.

Corollary 4. Let $f$ be a transcendental entire function of order of growth $\sigma(f)<1$ and $a \in \mathbb{C}$. Suppose that $\delta(a, f)>0$. Then $\Delta f$ has infinitely many fixed points.

\section{Some Lemmas}

Lemma 1 (lemma on the logarithmic derivative). Let $f(z)$ be a meromorphic function. If the function $f(z)$ has finite order, then

$$
m\left(r, \frac{f^{(k)}}{f}\right)=O(\log r)
$$

holds for any positive integer $k$.

Lemma 2 (see [18]). Let $f(z)$ be a meromorphic function with the exponent of convergence of poles $\lambda(1 / f)=\lambda<+\infty$ and let $c$ be a nonzero complex number. Then for each $\varepsilon>0$, we have

$$
N(r, f(z+c))=N(r, f)+O\left(r^{\lambda-1+\varepsilon}\right)+O(\log r) .
$$

Lemma 3. Let $f$ be a transcendental meromorphic function of order of growth $\sigma(f)<1$ and let $c$ be a nonzero complex number. Then

$$
N(r, f(z+c))=N(r, f)+O(\log r)
$$

Proof. Since the order $\sigma(f):=\sigma<1$, then $\lambda(1 / f)=\lambda \leq \sigma<$ 1. Therefore, for any $0<\varepsilon<1-\sigma$, it follows from Lemma 2 that

$$
\begin{aligned}
N(r, f(z+c)) & =N(r, f)+O\left(r^{\lambda-1+\varepsilon}\right)+O(\log r) \\
& =N(r, f)+O(1)+O(\log r) .
\end{aligned}
$$

That is,

$$
N(r, f(z+c))=N(r, f)+O(\log r)
$$

Lemma 4 (see [6]). Let $f$ be a function transcendental and meromorphic in the plane which satisfies

$$
\liminf _{r \rightarrow \infty} \frac{T(r, f)}{r}=0 .
$$

Then $\Delta f$ is transcendental.

Lemma 5. Let $f$ be a transcendental meromorphic function of order of growth $\sigma(f)=\sigma<1$. Then $\Delta f$ is transcendental.

Proof. Since the order $\sigma(f):=\sigma<1$, then, for any positive $\varepsilon(0<\varepsilon<1-\sigma)$, there exists $R>0$ such that for any $r>R$ we have

$$
T(r, f) \leq r^{\sigma+\varepsilon}
$$

Therefore,

$$
\liminf _{r \rightarrow \infty} \frac{T(r, f)}{r}=0
$$

\section{Lemma 5 follows Lemma 4.}

Lemma 6 (see [7]). Let $f(z)$ be a meromorphic function of finite order, then $\sigma(\Delta f) \leq \sigma(f)$.

Lemma 7 (see [7]). Let $f$ be a transcendental meromorphic function of order of growth $\sigma(f)<1$. Then for any $\varepsilon>0$ and any positive integer $k$, there exists a set $E \subset(1, \infty)$ that depends on $f$ and has finite logarithmic measure, such that for all $z$ satisfying $|z|=r \notin E \cup[0,1]$ we have

$$
\frac{\Delta^{k} f(z)}{f(z)}=\frac{f^{(k)}(z)}{f(z)}+O\left(r^{(k+1)(\sigma-1)+\varepsilon}\right) .
$$

It is easy to derive the following lemma from Lemma 1 and Lemma 7.

Lemma 8. Let $f$ be a transcendental meromorphic function of order of growth $\sigma(f)<1$. Then for any positive integer $k$ there exists a set $E \subset(1, \infty)$ that depends on $f$ and has finite logarithmic measure, such that

$$
m\left(r, \frac{\Delta^{k} f(z)}{f(z)}\right)=O(\log r), \quad r \notin E .
$$




\section{Proof of Theorems}

Proof. Since

$$
\frac{1}{f}=\frac{\Delta f}{z f}-\frac{z \Delta^{2} f-\Delta f}{z f} \frac{\Delta f-z}{z \Delta^{2} f-\Delta f},
$$

then

$$
\begin{aligned}
m\left(r, \frac{1}{f}\right) \leq & m\left(r, \frac{\Delta f}{z f}\right)+m\left(r, \frac{z \Delta^{2} f-\Delta f}{z f}\right) \\
& +m\left(r, \frac{\Delta f-z}{z \Delta^{2} f-\Delta f}\right)+O(1) \\
\leq & 2 m\left(r, \frac{\Delta f}{f}\right)+m\left(r, \frac{\Delta^{2} f}{f}\right) \\
& +m\left(r, \frac{\Delta f-z}{z \Delta^{2} f-\Delta f}\right)+O(\log r) .
\end{aligned}
$$

Applying the first fundamental theorem, we get

$$
\begin{aligned}
& m\left(r, \frac{1}{f}\right)=T(r, f)-N\left(r, \frac{1}{f}\right)+O(1) \\
& m\left(r, \frac{\Delta f-z}{z \Delta^{2} f-\Delta f}\right)= m\left(r, \frac{z \Delta^{2} f-\Delta f}{\Delta f-z}\right) \\
&+N\left(r, \frac{z \Delta^{2} f-\Delta f}{\Delta f-z}\right) \\
&-N\left(r, \frac{\Delta f-z}{z \Delta^{2} f-\Delta f}\right)+O(1) \\
& \leq m\left(r, \frac{z \Delta^{2} f-\Delta f}{\Delta f-z}\right) \\
&+N\left(r, \frac{z \Delta^{2} f-\Delta f}{\Delta f-z}\right)+O(1)
\end{aligned}
$$

Combining (14)-(15) we have

$$
\begin{aligned}
T(r, f) \leq & N\left(r, \frac{1}{f}\right)+2 m\left(r, \frac{\Delta f}{f}\right)+m\left(r, \frac{\Delta^{2} f}{f}\right) \\
& +m\left(r, \frac{z \Delta^{2} f-\Delta f}{\Delta f-z}\right) \\
& +N\left(r, \frac{z \Delta^{2} f-\Delta f}{\Delta f-z}\right)+O(\log r) \\
\leq & N\left(r, \frac{1}{f}\right)+N\left(r, \frac{1}{\Delta f-z}\right)+N\left(r, z \Delta^{2} f-\Delta f\right) \\
& +2 m\left(r, \frac{\Delta f}{f}\right) \\
& +m\left(r, \frac{\Delta^{2} f}{f}\right)+m\left(r, \frac{z \Delta^{2} f-\Delta f}{\Delta f-z}\right)+O(\log r) .
\end{aligned}
$$

Since

$$
\begin{aligned}
\Delta^{2} f & =\Delta(f(z+1)-f(z)) \\
& =f(z+2)-2 f(z+1)+f(z), \\
\Delta(\Delta f-z) & =\Delta(f(z+1)-f(z)-z) \\
& =f(z+2)-2 f(z+1)+f(z)-1,
\end{aligned}
$$

then, we can get

$$
\begin{aligned}
z \Delta^{2} f-\Delta f= & z f(z+2)-2 z f(z+1)+z f(z) \\
-f(z+1)+ & f(z) . \\
z \Delta(\Delta f-z)-(\Delta f-z)= & z f(z+2)-2 z f(z+1) \\
& +z f(z)-f(z+1)+f(z) .
\end{aligned}
$$

Therefore,

$$
\begin{aligned}
\frac{z \Delta^{2} f-\Delta f}{\Delta f-z} & =\frac{z \Delta(\Delta f-z)-(\Delta f-z)}{\Delta f-z} \\
& =\frac{z \Delta(\Delta f-z)}{\Delta f-z}-1, \\
N\left(r, z \Delta^{2} f-\Delta f\right) \leq & N(r, f(z+2))+N(r, f(z+1)) \\
& +N(r, f(z)) .
\end{aligned}
$$

Thus from Lemma 3 and (20), we deduce

$$
N\left(r, z \Delta^{2} f-\Delta f\right) \leq 3 N(r, f(z))+O(\log r) .
$$

By Lemmas 5 and 6, we know that $\Delta f-z$ is a transcendental meromorphic function of order of growth $\sigma(\Delta f-z) \leq$ $\sigma(f)<1$. It follows from Lemma 8 and (19) that there exists a set $E \subset(1, \infty)$ that has finite logarithmic measure, such that for any $r \notin E$ we have

$$
\begin{gathered}
m\left(r, \frac{\Delta f}{f}\right)=O(\log r), \\
m\left(r, \frac{\Delta^{2} f}{f}\right)=O(\log r), \\
m\left(r, \frac{z \Delta^{2} f-\Delta f}{\Delta f-z}\right)=O(\log r) .
\end{gathered}
$$

From (16) and (21)-(22), we have

$$
\begin{aligned}
T(r, f) \leq & 3 N(r, f)+N\left(r, \frac{1}{f}\right)+N\left(r, \frac{1}{\Delta f-z}\right) \\
& +O(\log r), \quad r \notin E .
\end{aligned}
$$


Denoting $g \equiv f-a$ by (23) we derive,

$$
\begin{aligned}
T(r, f) \leq & T(r, g)+O(1) \\
\leq & 3 N(r, g)+N\left(r, \frac{1}{g}\right)+N\left(r, \frac{1}{\Delta g-z}\right) \\
& +O(\log r) \\
\leq & 3 N(r, f)+N\left(r, \frac{1}{f-a}\right)+N\left(r, \frac{1}{\Delta f-z}\right) \\
& +O(\log r), \quad r \notin E .
\end{aligned}
$$

3.1. The Rest of the Proof of Theorem 1. By Lemma 6, we know that $\tau(\Delta f) \leq \sigma(f)$. If $\tau(\Delta f)<\sigma(f)$, by $\lambda(1 / f)<\sigma(f)$ and $\lambda(f, a)<\sigma(f)$, there exists a number $\eta<\sigma(f)$, such that for any sufficient $r$ we have

$$
\begin{gathered}
N(r, f)<r^{\eta}, \quad N\left(r, \frac{1}{f-a}\right)<r^{\eta}, \\
N\left(r, \frac{1}{\Delta f-z}\right)<r^{\eta} .
\end{gathered}
$$

Combining (24) and (25), we can get a contradiction. Therefore, we have $\tau(\Delta f)=\sigma(f)$.

3.2. The Rest of the Proof of Theorem 3. Since $\delta(\infty, f)=1$, then $N(r, f)=o(T(r, f))$. By $(24)$, we can get

$$
\begin{aligned}
(1-o(1)) T(r, f) \leq & N\left(r, \frac{1}{f-a}\right)+N\left(r, \frac{1}{\Delta f-z}\right) \\
& +O(\log r), \quad r \notin E .
\end{aligned}
$$

Since $\delta(a, f)>0$, then there is a positive number $\theta<1$ such that

$$
N\left(r, \frac{1}{f-a}\right)<\theta T(r, f) .
$$

If $\Delta f$ has only a finite number of fixed points, then from (26) and (27) we would have

$$
(1-o(1)-\theta) T(r, f) \leq O(\log r), \quad r \notin E .
$$

This contradicts $f$ being transcendental. Therefore, $\Delta f$ has infinitely many fixed points.

\section{Conflict of Interests}

The authors declare that there is no conflict of interests regarding the publication of this paper.

\section{Acknowledgments}

This research was partly supported by the National Natural Science Foundation of China (Grant nos. 11201395 and 61202313), by the Natural Science Foundation of Jiang$\mathrm{Xi}$ Province in China (Grant nos. 20132BAB211001 and 20122BAB201044) and by the Science Foundation of Educational Commission of Hubei Province (Grant nos. Q20132801 and D20132804).

\section{References}

[1] W. K. Hayman, Meromorphic Functions. Oxford Mathematical Monographs, Clarendon Press, Oxford, UK, 1964.

[2] L. Yang, Value Distribution Theory, Springer, Berlin, Germany; Science Press Beijing, Beijing, China, 1993.

[3] J. H. Zheng, Value Distribution of Meromorphic Functions, Tsinghua University Press, Beijing, China; Springer, Heidelberg, Germany, 2010.

[4] C. T. Chuang and C. C. Yang, Theory of Fix Points and Factorization of Mero- Morphic Functions, Mathematical Monogragh Series, Peking University Press, 1986.

[5] Z.-X. Chen and K. H. Shon, "Properties of differences of meromorphic functions," Czechoslovak Mathematical Journal, vol. 61, no. 1, pp. 213-224, 2011.

[6] W. Bergweiler and J. K. Langley, "Zeros of differences of meromorphic functions," Mathematical Proceedings of the Cambridge Philosophical Society, vol. 142, no. 1, pp. 133-147, 2007.

[7] Y. M. Chiang and S. J. Feng, "On the growth of logarithmic difference, difference equations and logarithmic derivatives of meromorphic functions," Transactions of the American Mathematical Society, vol. 361, pp. 3767-3791, 2009.

[8] R. G. Halburd and R. J. Korhonen, "Nevanlinna theory for the difference operator," Annales Academiae Scientiarum Fennicae Mathematica, vol. 31, pp. 463-478, 2006.

[9] R. G. Halburd and R. J. Korhonen, "Meromorphic solutions of difference equations, integrability and the discrete Painlevé equations," Journal of Physics A, vol. 40, no. 6, pp. R1-R38, 2007.

[10] R. G. Halburd and R. J. Korhonen, "Difference analogue of the lemma on the logarithmic derivative with applications to difference equations," Journal of Mathematical Analysis and Applications, vol. 314, pp. 477-487, 2006.

[11] N. Li and L. Z. Yang, "Value distribution of difference and qdifference polynomials," Advances in Difference Equations, vol. 2013, article 98, pp. 1-9, 2013.

[12] H. F. Liu and Z. Q. Mao, "On the meromorphic solutions of some linear difference equations," Advances in Difference Equations, vol. 2013, article 133, pp. 1-12, 2013.

[13] K. Liu and T. B. Cao, "Entire solutions of Fermat type qdifference differential equations," Electronic Journal of Differential Equations, vol. 2013, no. 59, pp. 1-10, 2013.

[14] K. Liu, H. Z. Cao, and T. B. Cao, "Entire solutions of Fermat type differential difference equations," Archiv Der Mathematik, vol. 99, pp. 147-155, 2012.

[15] H. Y. Xu, T. B. Cao, and B. X. Liu, "The growthof solutions of systems of complex q-shift difference equations," Advances in Difference Equations, vol. 2012, article 216, pp. 1-22, 2012.

[16] J. F. Xu and X. B. Zhang, "The zeros of q-shift difference polynomials of meromorphic functions," Advances in Difference Equations, vol. 2012, article 200, pp. 1-10, 2012.

[17] Z.-X. Chen and K. H. Shon, "Value distribution of meromorphic solutions of certain difference Painlevé equations," Journal of Mathematical Analysis and Applications, vol. 364, no. 2, pp. 556$566,2010$.

[18] Y.-M. Chiang and S.-J. Feng, "On the Nevanlinna characteristic of $\mathrm{f}(\mathrm{z}+\eta)$ and difference equations in the complex plane," Ramanujan Journal, vol. 16, no. 1, pp. 105-129, 2008. 


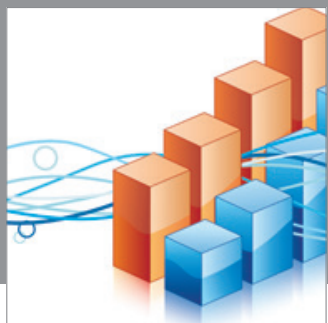

Advances in

Operations Research

mansans

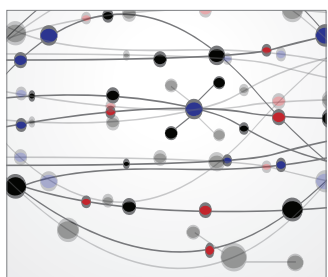

The Scientific World Journal
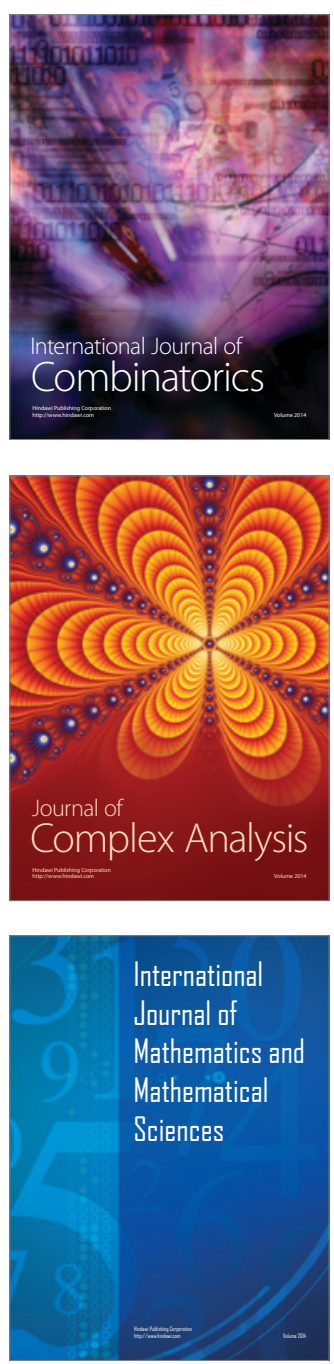
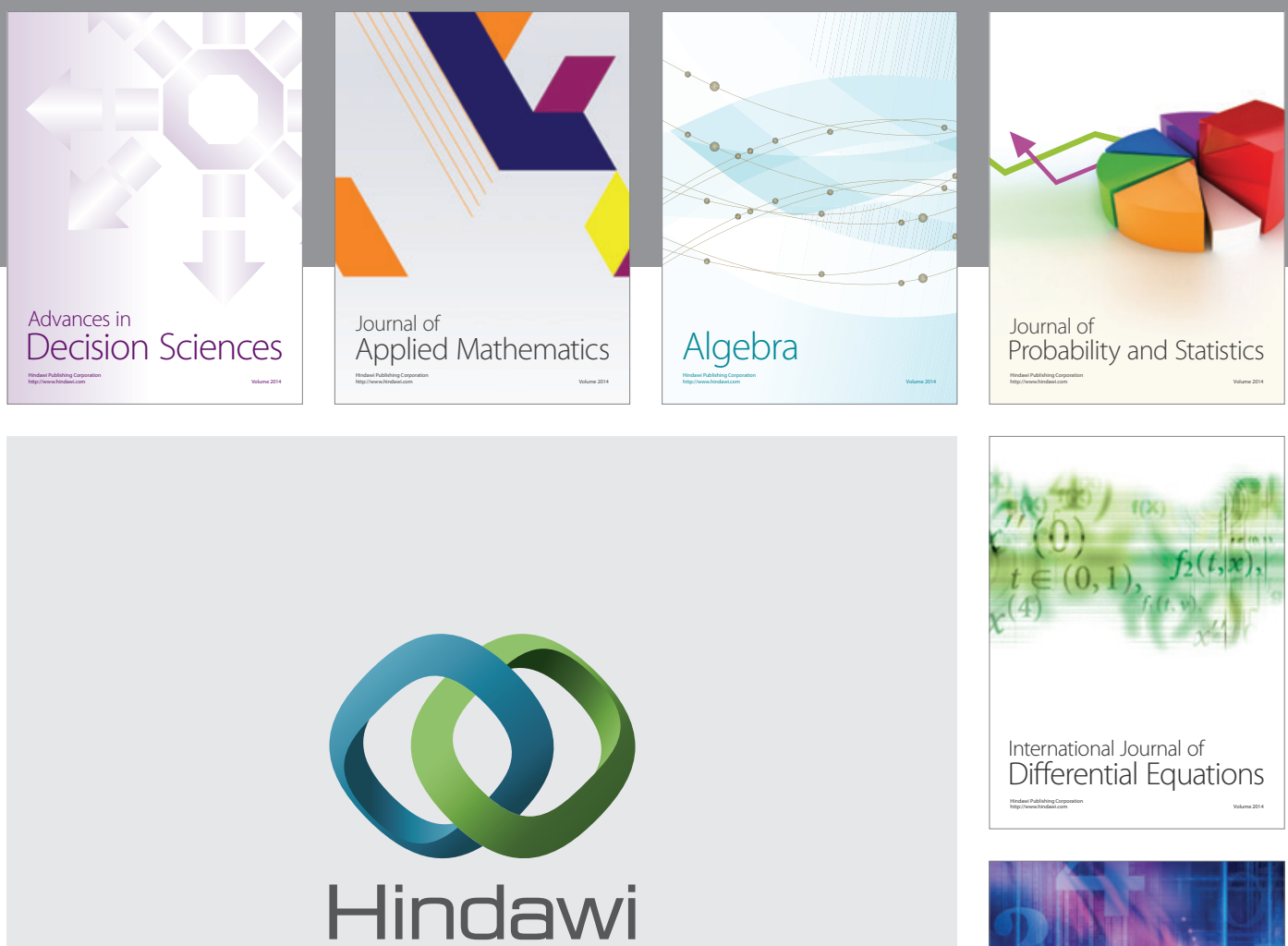

Submit your manuscripts at http://www.hindawi.com
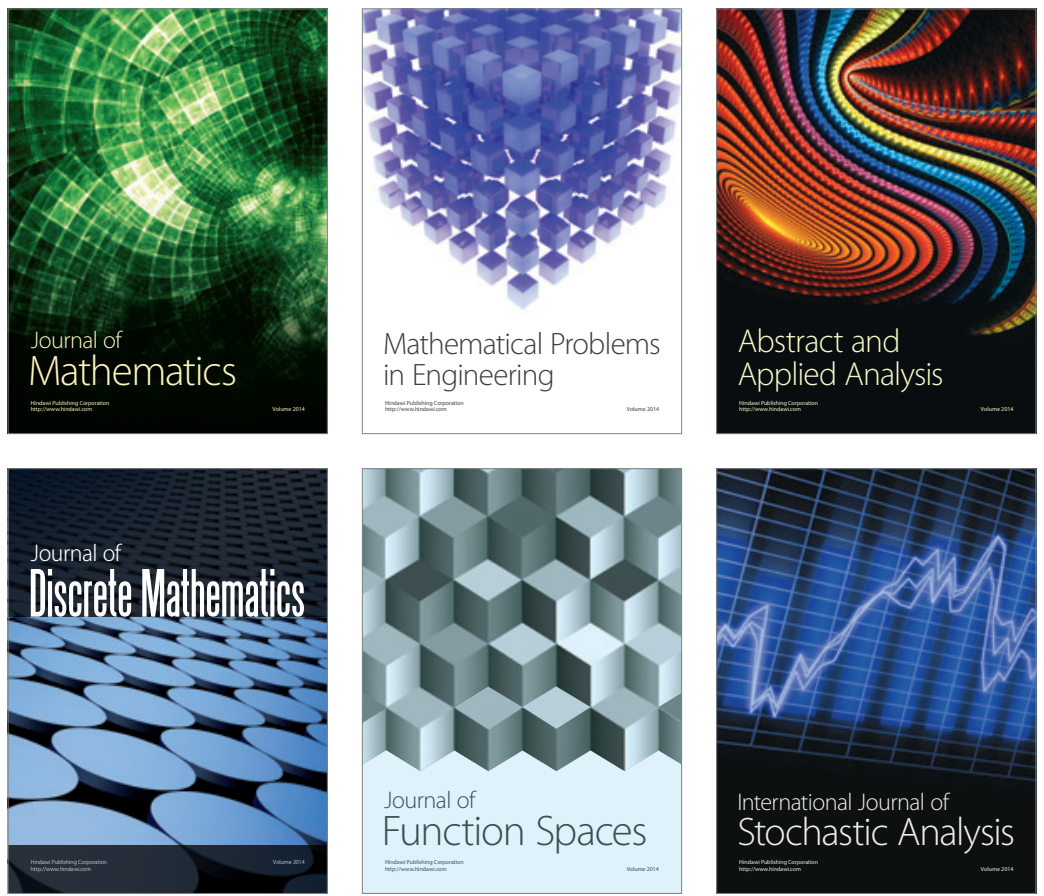

Journal of

Function Spaces

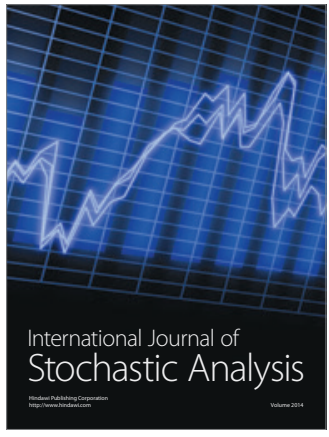

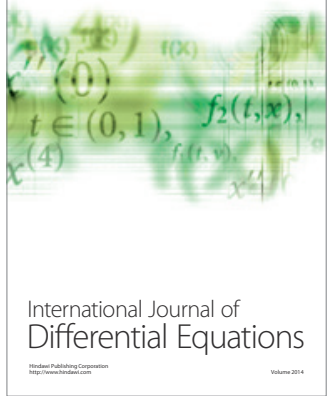
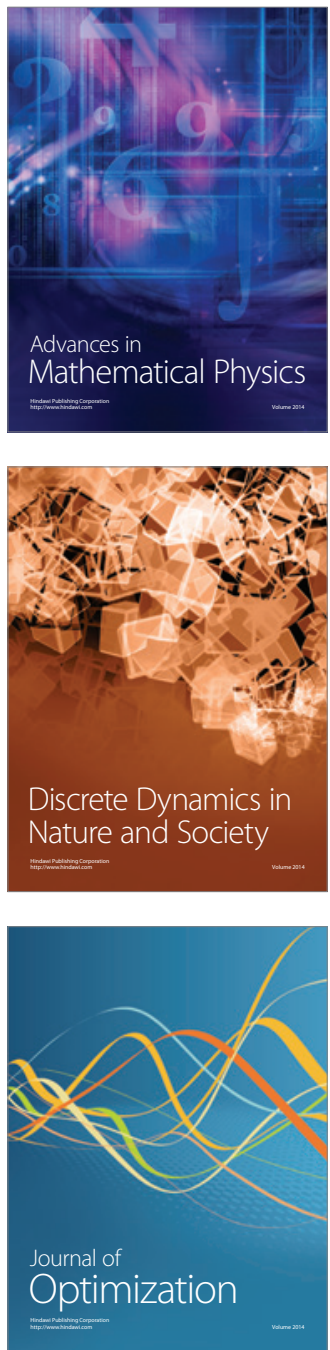